\title{
In-depth Study of Alkaline Fibroblast Growth Factor to Promote Effective Mechanism of Skin Wound Repair
}

\author{
Yun Yang \\ Plastic Surgery, Second Affiliated Hospital, Kunming Medical University Kunming, Yunnan, China, 650031
}

Keywords: basic fibroblast growth factor, skin wound, repair

\begin{abstract}
Basic fibroblast growth factor can promote angiogenesis and improve the healing rate of animals. As early as the 1990s, China began to study whether basic fibroblast growth factor has a role in promoting wound healing. The study found that basic fibroblast growth factor is a member of the fibroblast growth factor family, which is important for wound healing and tissue regeneration. Based on the wound healing process, the results of the study on the mechanism of basic fibroblast growth factor promoting wound healing were analyzed.
\end{abstract}

\section{Basic Fibroblast Growth Factor}

In the 1940s, Hoftman et al. found substances that increase the growth rate of fiber cells in brain and pituitary extracts. In the 1970s, this material was successfully isolated and purified and was named fibroblast growth factor. The study found that the isoelectric point of FGF is 9.6, so it is called basic fibroblast growth factor.

Basic fibroblast growth factor mainly exists in the pituitary, brain, nervous tissue, retina, placenta and other locations. Basic fibroblast growth factor mainly acts on fibroblasts, bone cells, skeletal muscle cells, etc., and its receptors are mainly distributed on the surface of these cells.

\section{Analysis of Wound Healing Process}

Wound healing is a complex physiological process involving cells, growth factors, extracellular matrix components, and proteases. Usually, we can divide the wound healing process into three stages: inflammation stage, proliferative stage and maturity stage. These three stages are continuous, and the three stages are briefly analyzed below.

\subsection{Inflammation Period}

After the wound appears in the human body, the first place to reach the wound is platelets, which release growth factors such as epidermal growth factor, insulin-like growth factor, and transforming growth factor. As these growth factors continue to spread, neutrophils and monocytes are attracted to growth factors and gradually reach the wound site. After that, monocytes will differentiate into macrophages, which can phagocytose bacteria and debris. After phagocytosis, macrophages can release Cuijin cell division and migration factors, which synthesize collagen and promote wound healing. Significance. Normally, the inflammatory period of a normal wound is 1 to 2 days.

\subsection{Proliferation Period}

As the second stage of wound healing, the proliferative phase will form granulation tissue and the initial blood vessels will occur. In short, granulation tissue is composed of fibroblasts, neovascularization, macrophages, fibronectin, collagen matrix, etc., and its main function is to compensate for the injured area. Compared with the inflammatory phase, the number of inflammatory cells is decreasing at this stage. The platelet-derived factor and transforming growth factor- $\beta$ are released from inflammatory cells, which will attract fibroblasts into the wound area and promote wound healing. Fibroblasts will migrate and spread when the wound appears in the human body for 3 to 4 days. After that, fibroblasts will release collagen and glycosaminoglycans, forming an amorphous gel in the wound. To form granulation tissue, the cells must form an extracellular matrix. 
Fibroblast proliferation and angiogenesis occur simultaneously during wound healing. Macrophages will form vascular endothelial growth factor and basic fibroblast growth factor, the main role of which is to promote the formation of blood vessels, which is of great significance for wound healing.

\subsection{Maturity}

Maturity is the final stage of wound healing, which is mainly from 3 weeks to 2 years after the appearance of the wound. As collagen is deposited, fibroblasts will continue to decrease. The study found that the intact skin of the human body is composed of type I and type III collagen, and the granulation tissue contains a large amount of type III collagen. At the same time, we also found that the newly formed collagen fibers at the wound site were randomly arranged without a certain order. Over time, collagen in the wound site gradually changed from type III to type I, and collagen fibers also began to be sorted in a certain order, which effectively increased the strength of skin tissue.

\section{Mechanism of Basic Fibroblast Growth Factor Promoting Wound Healing}

It can be seen from the above that the whole process of wound healing requires the participation of various cells such as keratinocytes, fibroblasts, endothelial cells, and platelets. These cells form new skin with growth factors, signaling pathways through proliferation, metastasis, and inflammatory responses. Among them, cell migration and proliferation are important for extracellular matrix formation and wound healing. In particular, the proliferation and migration of fibroblasts are important for the formation of granulation tissue and wound healing. In addition, the regeneration of blood vessels at the wound also has an important influence on wound healing. The newly formed blood vessels play an important role in promoting the formation of granulation tissue. Basic fibroblast growth factor is an important factor throughout the wound healing process.

Fu Xiaobing et al. studied 28 cases of 33 difficult to heal wounds. The study found that the healing rate of wounds treated by basic fibroblast growth factor was significantly improved, and most wounds healed within 4 weeks. It can be seen that basic fibroblast growth factor plays an important role in improving wound healing rate.

\subsection{Basic Fibroblast Growth Factor Promotes the Migration of Fibroblasts}

In 2010, Kanazawa et al. found that basic fibroblast growth factor promotes fibroblast metastasis by activating the PI3-Kinase-Rac1-JNK signaling pathway, which was observed by phalloidin staining in alkaline fibroblast growth. Under the action of factors, fibroblasts form lamellipodia, and basic fibroblast growth factor can promote the establishment of fibroblasts. Later, research on the mechanism by which basic fibroblast growth factor promotes fibroblast migration has continued. Xuan et al. used transcriptome sequencing technology to identify genes affected by basic fibroblast growth factor in fibroblasts and found a signaling pathway involved in regulation. The study confirmed that the process of fibroblast migration is NF- $\mathrm{BB}$. Many experts believe that the NF-кB-JNK signaling pathway is relatively independent and does not depend on the PI3K-Rac1-JNK pathway. NF-kB will be affected by basic fibroblast growth factor, which stimulates JNK phosphorylation to promote fibroblast migration.

Later, Wang et al. found that basic fibroblast growth factor activates the Wnt/ $\beta$-catenin signaling pathway in humans, prompting $\beta$-catenin to enter the nucleus, thereby activating the transcription factor TCF/LEF, and promoting FZD8 and Wnt3a. Gene transcription, while correctly feedback to increase basic fibroblast growth factor, promote fibroblast transfer. In addition, Wang $\mathrm{X}$ et al found that basic fibroblast growth factor regulated Wnt signaling pathway is related to PI3K/JNK pathway, which is because Wnt signaling pathway is located downstream of PI3K/JNK pathway. Zhu et al. proposed that the Hedgehog pathway is not only related to the PI3K-Rac1-JNK pathway, but also has an important relationship with the Wnt pathway. The specific process is: Hedgehog and Ptch1 cooperate, which plays an important role in activating Smo. Smo will activate PI3K-Rac1-JNK, which in turn will cause $\beta$-catenin to enter the nucleus. The $\beta$-catenin deposited in the nucleus will promote the transfer of fibroblasts by Smo, Gli1, Gli2 and the like. 


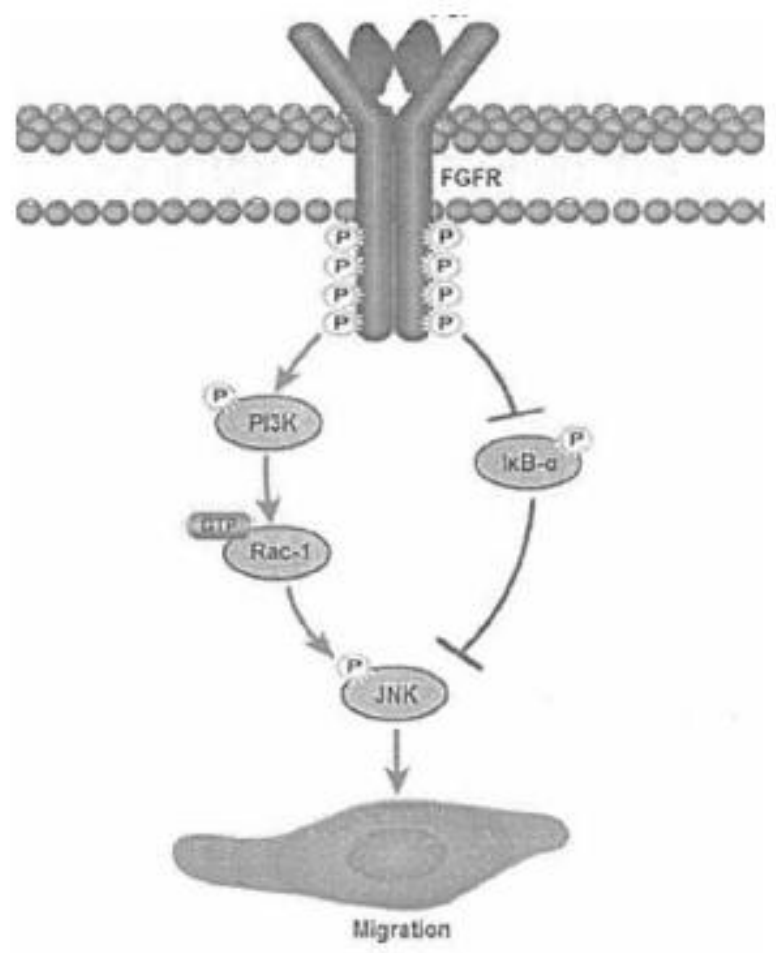

Figure 1. Basic Fibroblast Growth Factor Promotes Fibroblast Migration by Inhibiting the NF- $\mathrm{B}$ Pathway

\subsection{Basic Fibroblast Growth Factor Promotes Angiogenesis}

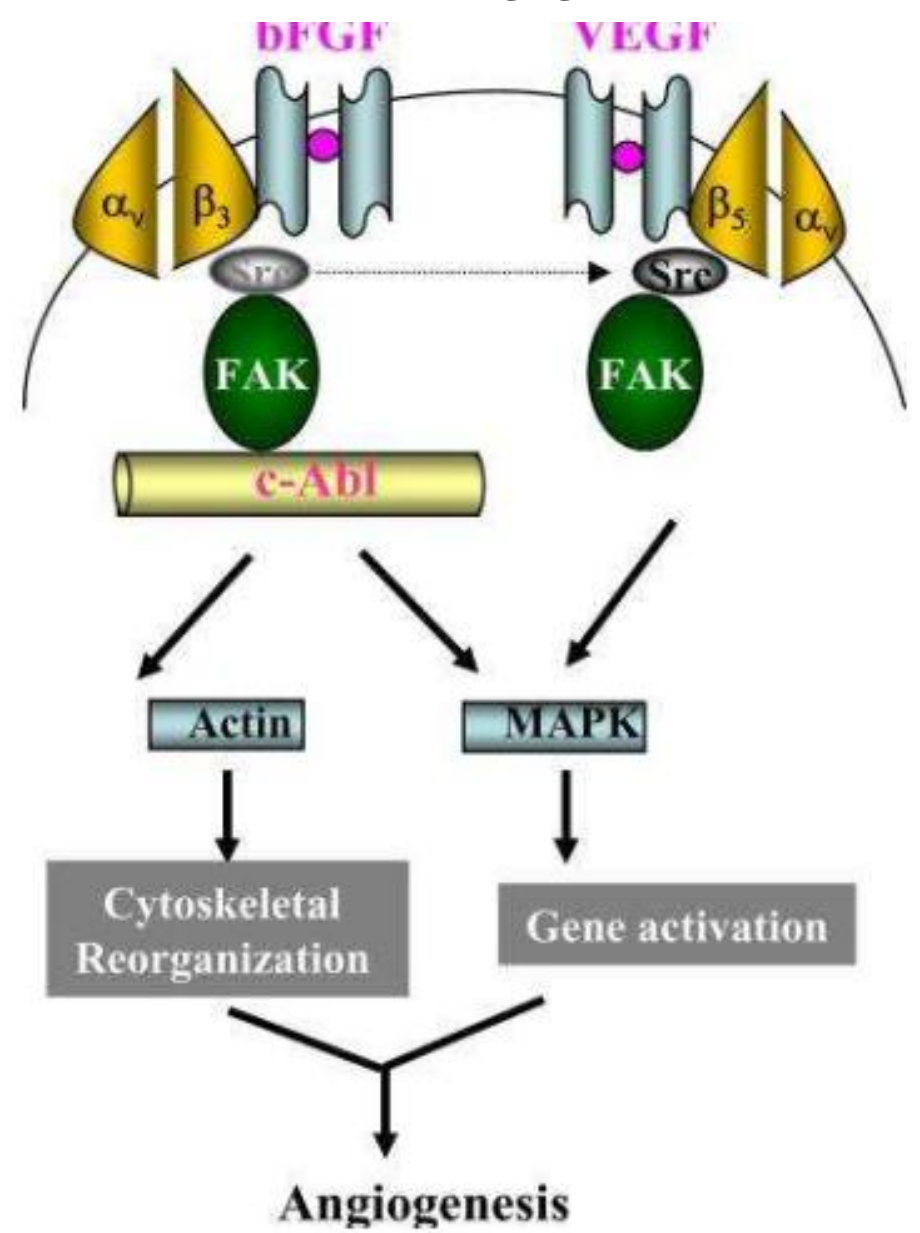

Figure 2. Mechanism of Basic Fibroblast Growth Factor Promoting Angiogenesis by Inducing Integrin $\alpha v \beta 3$ and FGF Receptors 
The formation of blood vessels is an important part of wound recovery. An important reason for the healing of diabetic foot is the small amount of angiogenesis. Studies have found that insufficient angiogenesis is an important factor affecting the rate of wound healing. Experiments have found that basic fibroblast growth factor plays an important role in increasing the rate of vascular endothelial cell formation. Yang Ting et al. found that the rat hindlimb arterial occlusion model was studied by studying vascular endothelial growth factor and basic fibroblast growth factor, compared with the study group using only vascular endothelial growth factor or basic fibroblast growth factor. The angiogenesis of the vascular endothelial growth factor and basic fibroblast growth factor study groups was good. Zhang Dongmei et al. constructed a rat model of vascular dementia, and found that basic fibroblast growth factor can penetrate the blood-brain barrier, increase the rate of vascular endothelial growth factor production, and promote angiogenesis. Yan et al. found that basic fibroblast growth factor promotes angiogenesis in a process that is different from vascular endothelial growth factor by studying tumor angiogenesis.

The study found that basic fibroblast growth factor can act on endothelial cells and endothelial cells, thereby achieving the goal of promoting angiogenesis. Endothelial progenitor cells belong to the premature cells of vascular endothelial cells, which are important for human angiogenesis. Studies have found that basic fibroblast growth factor can promote the proliferation and migration of endothelial progenitor cells. In the study, Tateishi-Yuyama et al found that the proliferation and migration function of diabetic endothelial progenitor cells can be restored when the basic fibroblast growth factor reaches a certain concentration.

\subsection{Basic Fibroblast Growth Factor Reduces Scar Formation}

If the wound is difficult to heal, a scar will form at the wound site. Usually, we can divide scars into hypertrophic scars and keloids. The main reason for the formation of these two scars is the excessive amount of immature collagen during remodeling. Hypertrophic scars will form red bulging scars at the wound site, and some of the scars will diminish or even disappear over time; keloids will extend beyond the wound site and such scars will not degenerate on their own. Basic fibroblast growth factor can increase the synthesis of MMP-1 and accelerate the degradation of collagen, so as to avoid hypertrophic scar formation due to excessive deposition of extracellular matrix.

Studies have found that keloids can occur if there are fewer epidermal stem cells and excessive proliferation of myofibroblasts. Epidermal stem cells are mostly located in the epidermis and hair basal layer hair follicles, and their proliferation and differentiation ability is large. Epidermal stem cells ensure normal skin structure and function, and epidermal stem cells can restore wounds by proliferation, migration and differentiation. Wang et al. proposed that Wang et al could activate the Notch1/Jagged1 pathway, promote epidermal stem cell proliferation, and ensure that it does not differentiate into myofibroblasts, thus achieving the goal of reducing scarring.

\section{Fibroblast Growth Factor Family}

The fibroblast growth factor family promotes fibroblast growth. The study found that there are 23 factors in the fibroblast growth factor family, and the factors that promote wound healing are: basic fibroblast growth factor, aFGF, FGF-9 and so on.

aFGF is important for promoting angiogenesis and wound repair. In the treatment of trauma and cardiovascular disease, a commonly used medicinal material is Salvia miltiorrhiza. It has been found that Salvia miltiorrhiza can promote aFGF production in human body, and then enhance the proliferation and differentiation speed of fibroblasts through its action on FGFR-3, thereby improving wound healing. speed. FGF-9 promotes wound healing through the Wnt / $\beta$-catenin signaling pathway. The study found that the expression of FGF-9 and FGF-9 mRNA was significantly enhanced after laser ablation of mouse skin or human skin. FGF-9 binding to FGFR-2 can effectively increase the amount of $\beta$-catenin into the nucleus. It is of great significance for wound healing. FGF-21 can increase the rate of glucose metabolism, which in turn increases the rate of wound healing. The main process is to increase the speed of wound healing by increasing the metabolic rate of cells and factors, and at the same time effectively inhibit inflammation. 
The fibroblast growth factor family is complementary in the process of wound healing, and it plays an important role in promoting wound healing by interacting with each other.

\section{Conclusion}

In summary, basic fibroblast growth factor plays an important role in improving the healing rate of wounds. During wound healing, it has an important relationship with cell proliferation, migration, angiogenesis and scar formation. Through research, we have found a signaling pathway related to basic fibroblast growth factor, and related drugs have been applied to clinical treatment. In order to improve the wound healing rate and therapeutic effect, we still need to strengthen the study of basic fibroblast growth factor-related pathways. With the development of science and technology, various treatment methods are constantly improving, and its application to clinical treatment can effectively shorten the treatment time.

\section{References}

[1] Lide Cui, Tiemin Li. Transplantation of Basic Fibroblast Growth Factor Gene-Modified Bone Marrow Mesenchymal Stem Cells to Repair Acute Kidney Injury[J]. Chinese Journal of Tissue Engineering Research, 2016, 20(28): 4169-4175.

[2] Yang Liu, Yilan Zhang, Yalan Huang, et al. Clinical Application of Artificial Dermis Combined with Basic Fibroblast Growth Factor in the Treatment of Scar and Deep Skin Wounds[J]. Chinese Journal of Burns, 2016, 32(4): 198- 203.

[3] Hailong $\mathrm{Xu}$, Yue Ding, Hong Xie, et al. Effects of Basic Fibroblast Growth Factor and Insulin-Like Growth Factor on Proliferation and Collagen Synthesis of Bone Marrow Mesenchymal Stem Cells [J]. Chinese Journal of Tissue Engineering Research, 2016, 20(6): 891-897.

[4] Xiaolu Jin, Yueying Zhu, Xiaojing Xu, et al. Study of Fibroblast Growth Factor in the Treatment of Chronic Refractory Wounds in the Elderly[J]. Journal of Nurses Training, 2017(18): 1650-1652.

[5] Xiao Wang, Jing Zhao, Mengying Du, et al. Experimental Study on Exogenous Basic Fibroblast Growth Factor-Induced Wound Healing in White Peony[J]. China Tissue Engineering Research, 2017, 21(34):5481- 5486.

[6] Heping Chang, Xu Li, Wei Wang, et al. Efficacy and Mechanism of Hyperbaric Oxygen Combined with Recombinant Human Basic Fibroblast Growth Factor in the Treatment of Skin-Healing Wounds[J]. China Pharmaceuticals, 2018(2): 46- 49. 